\title{
Dietary supplementation with cysteine prevents adverse metabolic outcomes of repeated cures with paracetamol in old rats
}

\author{
Carole Mast ${ }^{1}$, Charlène Pourpe ${ }^{1}$, Guillaume Voyard $^{2}$, Didier Rémond ${ }^{1}$, Carole Migné ${ }^{1}$, \\ Delphine Centeno ${ }^{1}$, Dominique Dardevet ${ }^{1}$, Isabelle Savary-Auzeloux ${ }^{1}$ and Isabelle Papet $^{1 *}$ \\ ${ }^{1}$ Institut National de la Recherche Agronomique (INRA), Unité de Nutrition Humaine (UNH), Plate-Forme d'Exploration du \\ Métabolisme (PFEM), Centre de Recherche en Nutrition Humaine (CRNH) Auvergne, Université Clermont Auvergne, F-6300O \\ Clermont-Ferrand, France \\ ${ }^{2}$ Institut de Chimie de Clermont-Ferrand, Université Clermont Auvergne, F-63000 Clermont-Ferrand, France
}

(Submitted 15 February 2017 - Final revision received 1 September 2017 - Accepted 20 September 2017)

\section{Abstract}

Cysteine (Cys), a conditionally indispensable amino acid, is required for the detoxification of paracetamol (acetaminophen, $N$-acetyl-paraaminophenol, 4-hydroxy-acetanilide, APAP), a drug of widespread use in older persons. We recently reported that repeated APAP cures could worsen sarcopenia in old rats, likely to be due to the impairment of Cys/GSH homoeostasis. The aim of the study was to evaluate whether a dietary Cys supplementation during APAP cures could improve Cys/GSH homoeostasis and thus preserve skeletal muscle. Male 21.5-monthold Wistar rats received three 2-week-long cures of APAP ( $1 \%$ of diet) alone or with extra Cys ( $0.5 \%$ of diet), intercalated with washout periods of 2 weeks (APAP and APAP-Cys groups, respectively). They were compared with untreated control rats (CT group). CT and APAPCys groups were pair-fed to the APAP group. Dietary Cys supplementation was efficient to prevent increase in liver mass $(P<0 \cdot 0001)$, decrease in liver GSH $(P<0 \cdot 0001)$, increase in blood GSH concentration $(P<0.0001)$, and to some extent, decrease in plasma free Cys concentration $(P<0.05)$, all induced by repeated APAP cures. The addition of Cys to APAP cures decreased plasma alanine transaminase $(P<0.05)$, the fractional synthesis rate of liver proteins $(P<0.01)$, and increased masses of extensor digitorum longus $(P<0.01)$, and soleus $(P<0.05)$, compared with the APAP group. Cys supplementation prevented alteration in Cys/GSH homoeostasis and increased some muscle masses in old rats under repeated cures with a non-toxic dose of APAP.

Key words: Cysteine: GSH: Liver: Skeletal muscles

Paracetamol (acetaminophen, $N$-acetyl-para-aminophenol, 4-hydroxy-acetanilide, APAP) is the most frequent analgesic and antipyretic drug of widespread use in older persons. As APAP is the large-scale first-line treatment of chronic pain of low to moderate intensity, it is often used over repeated cure periods $^{(1-3)}$. APAP detoxification initiates in the liver through phase I and II reactions ${ }^{(4,5)}$. Up to $90 \%$ of APAP is directly conjugated with sulphate (sulphation pathway) or glucuronide. In phase I, APAP is converted by cytochrome $\mathrm{P} 450$ into a highly reactive compound $N$-acetyl- $p$-benzoquinone imine (NAPQI). When the APAP dose increases, sulphation can become saturated then both glucuronide conjugation and, more significantly, oxidation to NAPQI, become higher. NAPQI is neutralised by GSH ( $\gamma$-glutamyl-cysteinyl-glycine), and then metabolised through the mercapturic acid pathway. The end products of APAP detoxification processes are excreted along with urine. Sulphate and GSH being both issued from cysteine
(Cys) ${ }^{(6)}$, APAP detoxification induces a net loss of this sulphur amino acid. Cys is provided by both dietary proteins and the breakdown of body proteins or GSH. Cys can also be endogenously synthesised from methionine and serine through the trans-sulphuration pathway. When the endogenous disposal of Cys is insufficient regarding its metabolic utilisations, Cys becomes an indispensable amino $\operatorname{acid}^{(6)}$. The net loss of sulphur to detoxify $3 \mathrm{~g}$ of APAP/d corresponded to $20 \%$ of the sulphur amino acids ingested by older patients eating $1 \mathrm{~g}$ of proteins $/ \mathrm{kg}$ body weight per $\mathrm{d}^{(7)}$. However, an arithmetic computation suggested that many APAP-treated older persons could be deficient in sulphur amino acids due to low dietary intakes $^{(8)}$.

The adverse outcome of APAP on Cys/GSH homoeostasis is documented $^{(9-11)}$, and depletion of liver GSH is the key player leading to hepatotoxicity in case of over dosage ${ }^{(12)}$. Within the therapeutic range, that is up to $4 \mathrm{~g} / \mathrm{d}$ for humans, APAP is

Abbreviations: APAP, paracetamol (acetaminophen, $N$-acetyl-para-aminophenol, 4-hydroxy-acetanilide); CT, control; Cys, cysteine; Cys-gly, cysteinyl-glycine; Hcy, homocysteine, GM, gastrocnemius.

* Corresponding author: I. Papet, fax +334736247 55, email isabelle.papet@inra.fr 
usually considered to be safe. However, a recent meta-analysis including eight observational studies highlighted increases in cardiovascular and gastrointestinal disorders and mortality with regular intake of therapeutic high doses ${ }^{(13)}$. It is also known that some individuals are sensitive to mild liver injury at therapeutic doses $^{(14-16)}$. Several cases of severe APAP-induced hepatotoxicity have also been reported with therapeutic doses ${ }^{(17-19)}$. These severe cases revealed a clear association of APAPinduced hepatotoxicity with fasting/undernutrition. This association was confirmed in an animal model mimicking undernourished patients, where protein-energy-restricted rats appeared to be more sensitive to APAP-induced hepatotoxicity than ad libitum fed rats ${ }^{(20)}$. These protein-energy-restricted rats also exhibited lower GSH levels under APAP, a key condition that can initiate hepatotoxicity ${ }^{(21)}$. Knowing that Cys is the ratelimiting substrate for GSH synthesis ${ }^{(22)}$; it could be considered that protein-energy-restricted rats under APAP suffer from a shortage of Cys.

Based on the mechanism of APAP-induced hepatotoxicity, the pharmacological molecule $N$-acetylcysteine (NAC) is the standard antidote which should be given as early as possible after poisoning ${ }^{(5,23,24)}$. The co-administration of APAP and NAC prevented toxicity associated with a single toxic dosing ${ }^{(25)}$. Nevertheless, NAC was found to be less efficient than a mixture of amino acids (Cys, methionine and serine) to protect the liver against a single administration of APAP in mice ${ }^{26)}$. Moreover, NAC did not prevent liver toxicity from chronic low-dose plus subacute high-dose paracetamol exposure in young or old mice $^{(27)}$. Further research is therefore required into how to alleviate the adverse metabolic outcomes of chronic treatments or repeated cures with APAP, notably for older persons.

The requirement in sulphur amino acids could be unachieved in elderly people chronically treated with APAP due to the low amount of food ingested. A dietary supplementation with Cys, the amino acid lost to ensure APAP detoxification, would be the most logical personalised nutritional strategy against the adverse metabolic outcomes of chronic treatments or repeated cures with APAP. Indeed, the APAP metabolism leads to extensive use of Cys that is definitively lost in the urine, thereby diverting Cys from its physiological uses. The adverse metabolic outcomes are not limited to the liver as they may also concern skeletal muscles. We recently published that non-toxic APAP treatments which decreased Cys/GSH availability for skeletal muscle, led to decreased muscle mass in adult rats ${ }^{(10)}$ and worsened sarcopenia (muscle loss linked to ageing) in old rats with suboptimal food intake ${ }^{(11)}$. It is well known that if availability of one amino acid alone is limited or its metabolic need is increased (e.g. Cys in sepsis or acute inflammation), protein synthesis is compromised especially in muscle ${ }^{(28)}$. Direct provision of Cys and its indirect supplies through cystine, GSH and other peptides containing Cys are indispensable for the muscle. In fact, Cys cannot be synthetised within the muscle because it lacks the enzymes necessary to synthetise Cys from methionine ${ }^{(29-31)}$. Dietary Cys supplementation should allow Cys/GSH homoeostasis to be maintained, thus avoiding any Cys shortage for muscles and therefore any adverse effects of APAP on skeletal muscle. Of note, increasing Cys content in the diet successfully restored liver GSH pools ${ }^{(32)}$ and limited weight loss and muscle wasting ${ }^{(33)}$ in septic rats. Dietary Cys supplementation has also been proven efficient in increasing (albeit weakly) Cys and GSH pools in ageing rats ${ }^{(34)}$. Therefore, the objective of the study was to evaluate whether dietary Cys supplementation could prevent the adverse outcomes of repeated APAP cures on Cys/GSH homoeostasis in old rats with suboptimal food intake, and consequently preserve their skeletal muscles.

\section{Methods}

\section{Animals and experimental design}

This study was performed in accordance with the current legislation on animal care and experimentation in France and received the approval (CE 08-13) of the local Ethical Committee, Comité d'Ethique en Matière d'Expérimentation Animale Auvergne. Male 20-21-month-old Wistar rats (Janvier Labs) were acclimatised 4 weeks before treatment in individual cages under standard conditions $\left(22 \pm 1^{\circ} \mathrm{C}, 12 \mathrm{~h}\right.$ light- $12 \mathrm{~h}$ dark cycle) with free access to water and the control diet (Table 1 ). At the end of this adaptation period, the rats were divided into three groups based on weight, food consumption and body composition. Body composition was assessed using MRI (Echo MRI International). In all, thirty-six rats (APAP group) were submitted to three cures (C1 to C3) of 2 weeks of APAP treatment, intercalated with washout periods (inter-cure, IC) of 2 weeks (Table 2). As previously explained, 'the cure model was chosen to reproduce the treatment of chronic pain in humans defined as daily pain lasting for at least 3 months ${ }^{(35)}$ and the alternation of painful and remission periods in patients. The length of 2 weeks for cures and IC took into account the difference between rat and human life expectancies, ${ }^{,(11)}$. In all, thirty-seven rats (APAP-Cys group) received the APAP-Cys diet instead of the APAP diet. The control group (CT, $n$ 36) received the control diet throughout the experimental period. The APAP-Cys and CT groups received a quantity of food adjusted to the real consumption of the APAP group throughout the experimental period. Food consumption was recorded daily and body weight twice a week.

Table 1. Composition of the experimental diets*

\begin{tabular}{lccc}
\hline Ingredients (g/kg DM) & Control diet & APAP diet & APAP-Cys diet \\
\hline Fish meal & $212 \cdot 0$ & $212 \cdot 0$ & $212 \cdot 0$ \\
Starch & $572 \cdot 4$ & $562 \cdot 4$ & $561 \cdot 1$ \\
Sucrose & 50 & 50 & 50 \\
Maltodextrin & 50 & 50 & 50 \\
Sunflower oil & $29 \cdot 4$ & $29 \cdot 4$ & $29 \cdot 4$ \\
Cellulose & 50 & 50 & 50 \\
Mineral mixture AIN-93 & 20 & 20 & 20 \\
Vitamin mixture AIN-93 & 10 & 10 & 10 \\
Choline bitartrate & $2 \cdot 5$ & $2 \cdot 5$ & 2.5 \\
APAP & - & 10 & 10 \\
Ala & $3 \cdot 7$ & $3 \cdot 7$ & - \\
Cys & - & - & 5.0 \\
Food dye E124 & - & Trace & - \\
Food dye E133 & Trace & - & - \\
\hline
\end{tabular}

APAP, paracetamol (acetaminophen, $\mathrm{N}$-acetyl-para-aminophenol, 4-hydroxy-acetanilide); APAP-Cys, paracetamol-cysteine.

* The composition of the control diet was $16 \%$ proteins, $68 \%$ carbohydrates, $6 \%$ fat, $5 \%$ fibre and $5 \%$ others. 
Cysteine effect in old rats under paracetamol

Table 2. Distribution of the diets over the experimental periods according to the experimental group*

\begin{tabular}{lcccc}
\hline Experimental periods & C1 & IC1 & C2 & IC2 \\
\hline CT group & Control diet & Control diet & Control diet & Control diet \\
APAP group & APAP diet & Control diet & APAP diet & Control diet \\
APAP-Cys group & APAP-Cys diet & Control diet & APAP-Cys diet & Control diet
\end{tabular}

C, cure; IC, inter-cure; CT, control; APAP, paracetamol (acetaminophen, $\mathrm{N}$-acetyl-para-aminophenol, 4-hydroxy-acetanilide); APAP-Cys, paracetamol-cysteine.

* APAP-Cys and CT groups were pair-fed to the APAP group. Each C or IC lasted 2 weeks.

\section{Diets}

Repeated APAP cures were performed as previously described, the APAP powder (Sigma-Aldrich) being mixed with other ingredients $^{(11)}$ (Table 1). Food dyes were added to diets for identification purposes and to avoid any confusion during feeding periods. The dietary level of APAP ( $1 \% \mathrm{w} / \mathrm{w})$ was chosen as an equivalent dose of $4 \mathrm{~g} / \mathrm{d}$ for humans ${ }^{(10)}$. Consumption of the $1 \%$ APAP diet was expected to provide a daily dose within the range of the typical anti-nociceptive doses $\left(200-300 \mathrm{mg} / \mathrm{kg}\right.$ ) for rats ${ }^{(36)}$. L-Cys (Sigma-Aldrich) was also mixed with the ingredients to prepare the APAP-Cys diet. The Cys supplement was calculated to compensate for APAPinduced sulphur urinary loss on the basis that $54 \%$ of APAP molecules was conjugated with sulphate or GSH in our previous study ${ }^{(10)}$. Taking into account molar masses of APAP and Cys, the $1 \%$ APAP diet was supplemented with $0.5 \%(\mathrm{w} / \mathrm{w})$ of L-Cys. The equivalent Cys dosage for humans would be about $2 \mathrm{~g} / \mathrm{d}$, an intake within the nutritional range, thus expected to be safe. The APAP and control diets were supplemented with L-alanine to make them iso-nitrogenous to the APAP-Cys diet.

\section{In vivo protein synthesis}

Rates of protein synthesis were measured in the liver and muscle at the end of the third cure using the flooding dose method as previously described ${ }^{(11)}$. After an overnight fast, $\left[1-{ }^{13} \mathrm{C}\right]$ valine (Cambridge Isotope Laboratories) (98.6\%, $150 \mu$ moles $/ 100 \mathrm{~g}$ body weight) was injected into a lateral tail vein $25 \mathrm{~min}$ before euthanasia ( $50 \mathrm{mg}$ pentobarbital/kg body weight, intra peritoneal) to flood the precursor pool for protein synthesis. Blood was sampled from the aorta, plasma was separated by centrifugation at $2000 \boldsymbol{g}$ for $15 \mathrm{~min}$ at $4^{\circ} \mathrm{C}$, and immediately frozen in liquid $\mathrm{N}_{2}$. The liver was immediately removed, washed with saline and weighed. Skeletal muscles: gastrocnemius (GM), tibialis anterior (TA), soleus (SOL) and extensor digitorum longus (EDL) were carefully dissected from the left posterior leg and weighed. Immediately after weighing, the liver and GM were frozen in liquid $\mathrm{N}_{2}$. The GM was chosen for protein synthesis measurements because of its mixedto-slow fibre type and its size allowing multiple assays once milled. All frozen samples were stored at $-80^{\circ} \mathrm{C}$ before analyses. Frozen tissues were finely pulverised in liquid $\mathrm{N}_{2}$ using a ball mill (Dangoumeau) before analyses.

Free and protein-bound valine enrichments were determined by mass spectrometer and protein quantified using the bicinchoninic acid method, as previously described ${ }^{(37)}$. Fractional and absolute synthesis rates were calculated, as previously described ${ }^{(11)}$.

\section{GSH and other aminothiols}

Total free GSH (GSH, GSSG and other small disulphides) concentration was quantified in the liver, GM and blood with an automated analyzer (ABX Pentra 400; Horiba) using a standard enzymatic recycling procedure and 5,5'- dithio-bis-2-nitrobenzoic acid (Ellman reagent) as oxidant, as previously described ${ }^{(38)}$.

Plasma concentrations of free and protein-bound aminothiols (Cys, GSH, $\gamma$-glutamyl-cysteine ( $\gamma$-Glu-Cys), cysteinyl-glycine (Cys-gly) and homocysteine (Hcy) were quantified by reversedphase HPLC, as previously described ${ }^{(11)}$. In fact, protein-bound $\gamma$-Glu-Cys was too low to be accurately assessed.

\section{Hepatotoxicity and inflammatory markers}

Hepatotoxicity was assessed by measurement of plasma alanine transaminase (ALT) and aspartate transaminase (AST) activities by photometry using an automated analyzer (ABX Pentra 400) and test kits A11A01629 and A11A01627 (Horiba), respectively. Plasma acute phase protein ( $\alpha_{2}$-macroglobulin, fibrinogen and albumin) concentrations were quantified as previously described ${ }^{(39)}$.

\section{Statistical analysis}

Group size was based on our previous results ${ }^{(11)}$ to allow for the detection of differences for GM mass with a sufficient power of $80 \%$ at the level of significance of $0 \cdot 05$. Results are expressed as means with their standard errors. Food intake and body weight were analysed using ANOVA for repeated measures with time as the within-rat factor and group as the second variable. The significance of differences was further analysed by Ryan-Einot-Gabriel-Welch $q$ (REGWQ) test. Endpoint results were analysed using the one-way ANOVA, followed by the Tukey test. Analyses were performed using XLSTAT for Windows, version 2013.1.01 software (Addinsoft) and the significance was set at $P \leq 0 \cdot 05$.

\section{Results}

\section{Daily food intake and body weight}

During the adaptation period, there was no significant difference in the food intake and body weight between groups (Table 3). Whatever the experimental period, food intakes were lower than during the adaptation period. As the CT and APAP-Cys groups were pair-fed to the APAP group, daily food intake of the three groups matched perfectly during cures and IC (no group effect or interaction with time effect). The daily APAP consumption during the cures was about $300 \mathrm{mg} / \mathrm{kg}$ per d. Body weight decreased 
Table 3. Food intake, body weight and lean mass over the experimental periods in control (CT), paracetamol (acetaminophen, $N$-acetyl-para-aminophenol, 4-hydroxy-acetanilide, APAP) and paracetamol-cysteine (APAP-Cys) treated groups*

(Mean values with their standard errors)

\begin{tabular}{|c|c|c|c|c|c|c|c|c|c|c|c|c|c|}
\hline & \multicolumn{2}{|c|}{ Adaptation } & \multicolumn{2}{|c|}{$\mathrm{C} 1$} & \multicolumn{2}{|c|}{ IC1 } & \multicolumn{2}{|c|}{$\mathrm{C} 2$} & \multicolumn{2}{|c|}{ IC2 } & \multicolumn{3}{|c|}{$\mathrm{C} 3$} \\
\hline & Mean & SEM & Mean & SEM & Mean & SEM & Mean & SEM & Mean & SEM & Mean & & SEM \\
\hline \multicolumn{14}{|l|}{ Food intake $(\mathrm{g} / \mathrm{d})$} \\
\hline CT & $22 \cdot 0$ & 0.6 & $18 \cdot 8$ & 0.1 & $19 \cdot 5$ & 0.2 & $19 \cdot 7$ & 0.2 & $19 \cdot 8$ & 0.2 & $20 \cdot 0$ & & 0.2 \\
\hline APAP & $22 \cdot 4$ & 0.5 & $19 \cdot 3$ & 0.4 & $20 \cdot 2$ & 0.3 & $20 \cdot 1$ & 0.3 & $19 \cdot 9$ & 0.3 & 20.5 & & 0.3 \\
\hline $\begin{array}{l}\text { APAP-Cys } \\
\text { Global time effect }\end{array}$ & \multicolumn{2}{|c|}{$21 \cdot 2$ a } & \multicolumn{2}{|c|}{$\mathrm{b}$} & \multicolumn{2}{|l|}{20.1} & \multicolumn{2}{|c|}{$\mathrm{c}$} & \multicolumn{2}{|c|}{$\mathrm{c}$} & \multicolumn{3}{|c|}{$\mathrm{c}$} \\
\hline \multicolumn{14}{|c|}{ Body weight at the end of each period $(\mathrm{g})$} \\
\hline CT & 701 & 13 & 680 & 12 & 672 & 11 & 668 & 10 & 660 & 10 & 644 & & 9 \\
\hline APAP & 684 & 10 & 668 & 10 & 667 & 10 & 659 & 10 & 658 & 10 & 643 & & 10 \\
\hline $\begin{array}{l}\text { APAP-Cys } \\
\text { Global time effect }\end{array}$ & 694 & 12 & 675 & 11 & 670 & 10 & 664 & 10 & 656 & 9 & 640 & c & 9 \\
\hline
\end{tabular}

C, cure; IC, inter-cure.

a,b,c Values with unlike superscript letters were significantly different $(P<0.05)$ within time points (REGWQ test).

${ }^{*}$ ANOVA for repeated measurements with time as the within-rat factor and group as the second variable for food intake: time: $P<0 \cdot 0001$, group: $P=0.120$, time $\times$ group: $P=0.293$; body weight: time: $P<0.0001$, group: $P=0.869$, time $\times$ group: $P<0.001$.
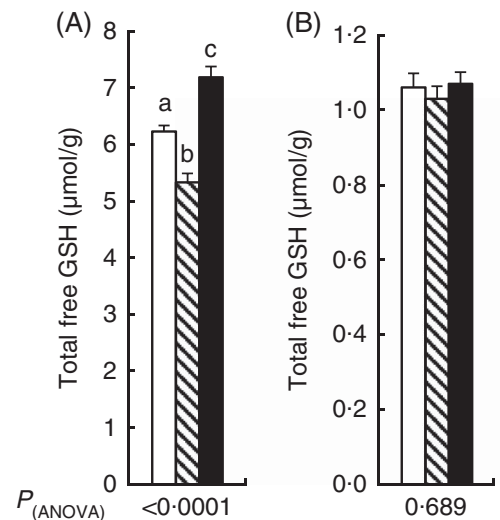

(C)

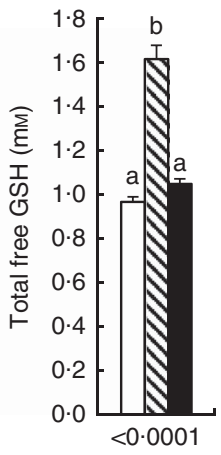

Fig. 1. Total free GSH (GSH, GSSG and other small disulphides) concentration in liver $(A)$, gastrocnemius muscle $(B)$ and blood $(C)$ of control, paracetamol and paracetamol-cysteine treated groups. $\square$, control group; $\mathbf{\nabla}$, paracetamol group (acetaminophen, $\mathrm{N}$-acetyl-para-aminophenol, 4-hydroxy-acetanilide, APAP); $\mathbf{\square}$, APAP-cysteine group. ${ }^{a, b, c}$ Mean values with unlike letters were significantly different (ANOVA followed by the Tukey test, $P<0.05$ ).

throughout the experiment in all groups with no group effect, the overall body weight loss being about $7 \%$ (Table 3 ).

\section{Tissue GSH}

Total free GSH concentration in the liver at the end of the experiment was $14 \%$ lower in the APAP group compared with the CT group (Fig. 1(A)). Liver GSH was higher in the APAPCys group than the APAP and CT groups by 35 and $15 \%$, respectively. There was no significant difference in GM concentration in total GSH between the three groups (Fig. 1(B)). Total blood GSH concentration was $67 \%$ higher in the APAP group than the CT group. The addition of Cys to APAP cures normalised blood GSH (Fig. 1(C)).

\section{Plasma aminothiols}

Compared with CT, the plasma concentration of free Cys (Cys + cystine + small Cys disulphides) decreased by $10 \%$ with repeated
(A)
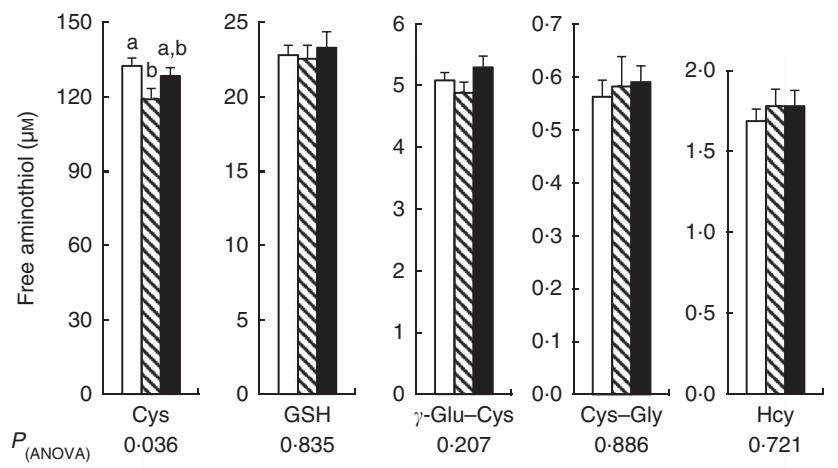

(B)
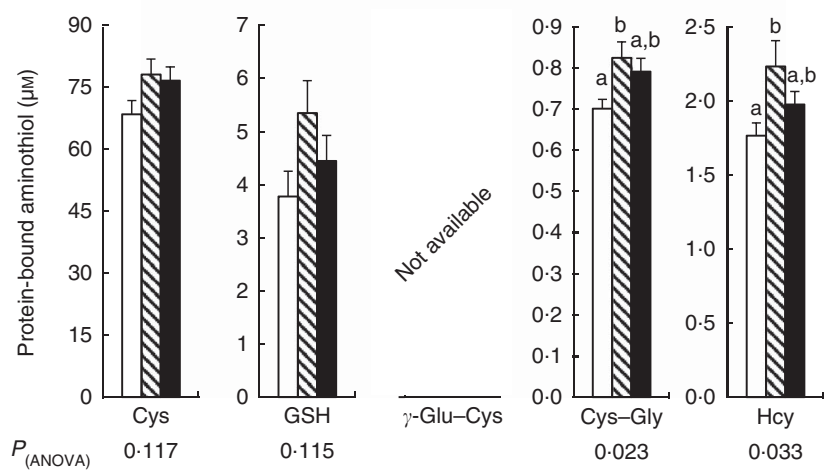

Fig. 2. Plasma free $(A)$ and protein-bound $(B)$ aminothiols in control, paracetamol and paracetamol-cysteine treated groups. $\square$, control group; $\mathbf{\nabla}$, paracetamol group (acetaminophen, $\mathrm{N}$-acetyl-para-aminophenol, 4-hydroxy-acetanilide, APAP); $\square$, APAP-cysteine (Cys) group; Cys, cysteine; Cys-gly, cysteinylglycine; $\gamma$-Glu-Cys, $\gamma$-glutamyl-cysteine; Hcy, homocysteine. ${ }^{\text {a,b }}$ Mean values with unlike letters were significantly different (ANOVA followed by the Tukey test, $P<0.05)$.

cures with APAP alone (Fig. 2(A)). Plasma free Cys of the APAPCys group did not differ significantly from the other two groups. There was no significant difference in plasma concentrations of the other free aminothiols (GSH, $\gamma$-Glu-Cys, Cys-Gly and Hcy) between the three groups. Compared with CT, the plasma 
Table 4. Liver and muscle masses in control (CT), paracetamol (acetaminophen, N-acetyl-para-aminophenol, 4-hydroxy-acetanilide, APAP) and paracetamol-cysteine (APAP-Cys) treated groups

(Mean values with their standard errors)

\begin{tabular}{|c|c|c|c|c|c|c|c|c|c|c|}
\hline & \multicolumn{2}{|c|}{ Liver (g) } & \multicolumn{2}{|c|}{ GM (g) } & \multicolumn{2}{|c|}{$\mathrm{TA}(\mathrm{mg})$} & \multicolumn{2}{|c|}{$\mathrm{EDL}(\mathrm{mg})$} & \multicolumn{2}{|c|}{$\mathrm{SOL}(\mathrm{mg})$} \\
\hline & Mean & SEM & Mean & SEM & Mean & SEM & Mean & SEM & Mean & SEM \\
\hline CT & $16 \cdot 8^{a}$ & 0.4 & 2.01 & 0.08 & 625 & 23 & $199^{a, b}$ & 7 & $205^{a, b}$ & 8 \\
\hline APAP & $19 \cdot 3^{b}$ & 0.4 & 2.02 & 0.08 & 610 & 25 & $179^{a}$ & 6 & $186^{a}$ & 8 \\
\hline APAP-Cys & $17 \cdot 6^{a}$ & 0.3 & 2.04 & 0.07 & 620 & 23 & $207^{b}$ & 6 & $215^{b}$ & 7 \\
\hline$P_{\text {(ANOVA) }}$ & \multicolumn{2}{|c|}{$<0.0001$} & \multicolumn{2}{|c|}{0.958} & \multicolumn{2}{|c|}{0.902} & \multicolumn{2}{|c|}{0.007} & \multicolumn{2}{|c|}{0.032} \\
\hline
\end{tabular}

GM, gastrocnemius; TA, tibialis anterior; EDL, extensor digitorum longus; SOL, soleus.

a,b Mean values within a column with unlike superscript letters were significantly different $(P<0.05$, ANOVA followed by the Tukey test).

concentrations of protein-bound Cys-Gly and Hcy were 18 and $27 \%$ higher in the APAP group than the CT group, respectively (Fig. 2(B)). Plasma protein-bound Cys-Gly and Hcy of the APAPCys group did not differ significantly from the other two groups. There was no significant difference in plasma concentrations of protein-bound Cys and GSH between the three groups.

\section{Tissue masses, protein contents and synthesis rates}

Liver mass was $15 \%$ higher in the APAP group compared with the CT group (Table 4). The addition of Cys to APAP cures normalised the liver mass. There was no significant difference in the protein contents of the liver between the three groups (Fig. 3(A)). Amongst the three groups, there was only a difference in the fractional synthesis rate of liver proteins between APAP-Cys and APAP groups, with an $8 \%$ decrease induced by the addition of Cys to the APAP cures (Fig. 3(A)). Despite a significant group effect on the absolute synthesis rate of liver proteins, post-ANOVA tests reveal no significant effect between groups when compared with each other (Fig. 3(A)).

There was no significant difference in masses of GM and TA between the three groups and the masses of EDL and SOL of the CT group did not significantly differ from the APAP and APAP-Cys groups (Table 4). However, the addition of Cys to APAP cures increased EDL and SOL masses by $16 \%$ each. There was no significant difference in the protein content of GM or the fractional and absolute synthesis rates of GM proteins between the three groups (Fig. 3(B)).

\section{Hepatotoxicity and inflammation}

Amongst the three groups, the activity of ALT only differed between the APAP-Cys and APAP groups; with a $30 \%$ decrease induced by the addition of Cys to the APAP cures (Table 5). There was no significant difference in plasma AST activity or plasma concentrations of $\alpha_{2}$-macroglobulin and fibrinogen between the three groups (Table 5). Plasma albumin concentration was $13 \%$ higher in the APAP-Cys group than the CT group (Table 5).

\section{Discussion}

Adverse metabolic outcomes occur in old rats submitted to repeated cures with a $1 \% \mathrm{APAP} \operatorname{diet}^{(11)}$. In the present
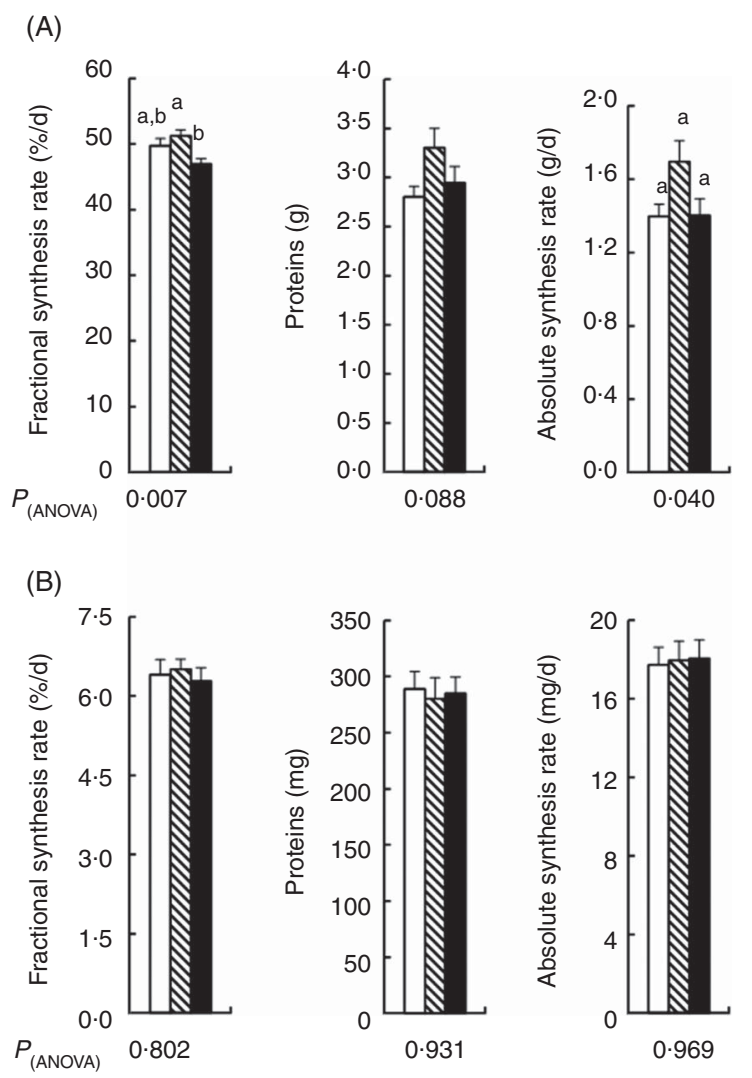

Fig. 3. Fractional synthesis rate, protein content and absolute synthesis rate in the liver (A) and gastrocnemius muscle (B) of control ( $\square$ ), paracetamol (acetaminophen, $\mathrm{N}$-acetyl-para-aminophenol, 4-hydroxy-acetanilide, APAP, $\mathrm{\nabla}$ ) and paracetamol-cysteine (APAP-Cys, $\square$ ) treated groups. ${ }^{a, b}$ Mean values with unlike letters were significantly different (ANOVA followed by the Tukey test, $P<0.05$ )

experiment, the addition of Cys to the APAP cures, at a level equivalent to the detoxification needs, was efficient to prevent all APAP-induced adverse outcomes for the liver where detoxification takes place, but also blood GSH, and to some extent, plasma free Cys and protein-bound Cys-Gly and Hcy. These results were obtained in old rats with suboptimal food intake under repeated cures with a non-toxic APAP dosage an animal model of APAP-treated older persons.

Liver GSH data extend previous observations made by others in growing or adult mice under chronic treatment with $\mathrm{APAP}^{(9,40)}$. In these studies, providing methionine largely above 
Table 5. Transaminase activities and acute phase proteins in the plasma of control (CT), paracetamol (acetaminophen, $N$-acetyl-para-aminophenol, 4-hydroxy-acetanilide, APAP) and paracetamol-cysteine (APAP-Cys) treated groups

(Mean values with their standard errors)

\begin{tabular}{|c|c|c|c|c|c|c|c|c|c|c|}
\hline & \multicolumn{2}{|c|}{ ALT (IU/I) } & \multicolumn{2}{|c|}{ AST (IU/I) } & \multicolumn{2}{|c|}{$a_{2}$-Macroglobulin (mg/l) } & \multicolumn{2}{|c|}{ Fibrinogen (g/l) } & \multicolumn{2}{|c|}{ Albumin (g/l) } \\
\hline & Mean & SEM & Mean & SEM & Mean & SEM & Mean & SEM & Mean & SEM \\
\hline CT & $45 \cdot 4^{\mathrm{a}, \mathrm{b}}$ & $2 \cdot 4$ & $90 \cdot 0$ & 4.4 & $12 \cdot 9$ & 1.5 & $3 \cdot 16$ & 0.15 & $16 \cdot 3^{a}$ & 0.5 \\
\hline APAP & $62 \cdot 3^{a}$ & $8 \cdot 6$ & 97.9 & $12 \cdot 0$ & 11.6 & 1.8 & 2.92 & 0.13 & $17 \cdot 3^{a, b}$ & 0.5 \\
\hline APAP_Cys & $43.9^{b}$ & 1.7 & 85.6 & 3.4 & $12 \cdot 7$ & 4.4 & 2.83 & 0.15 & $18 \cdot 4^{\mathrm{b}}$ & 0.4 \\
\hline$P_{\text {(ANOVA) }}$ & \multicolumn{2}{|c|}{0.021} & \multicolumn{2}{|c|}{0.505} & \multicolumn{2}{|c|}{0.944} & \multicolumn{2}{|c|}{0.249} & \multicolumn{2}{|c|}{0.008} \\
\hline
\end{tabular}

ALT, alanine transaminase; AST, aspartate transaminase.

${ }_{\mathrm{a}, \mathrm{b}}$ Mean values within a column with unlike superscript letters were significantly different $(P<0.05$, ANOVA followed by the Tukey test).

the requirement level (up to $1 \%$ of the $\operatorname{diet}(\mathrm{w} / \mathrm{w})$ for methionine as well as for APAP) prevented APAP-induced decreases in hepatic GSH. Cys supplementation is presently efficient, whereas methionine is not synthesisable from Cys. Thus, the beneficial effect of methionine on liver GSH is likely to have resulted from its capacity to provide Cys, meaning that the endogenous synthesis of Cys from methionine and serine was insufficient to maintain liver GSH when APAP-treated animals received a standard diet. Methionine is the indispensable sulphur amino acid, whose overload may have adverse cardiovascular effects directly or through an accumulation of $\mathrm{Hcy}^{(41,42)}$. Cys is a conditionally indispensable amino acid because its endogenous synthesis can become insufficient to meet its metabolic utilisations in some circumstances ${ }^{(6)}$. Altogether, the requirement of sulphur amino acids is increased by APAP and Cys can fulfil this demand.

The paradoxical large APAP-induced increase in blood GSH concentration confirms our previous observation in adult rats treated for $17 \mathrm{~d}$ with $\operatorname{APAP}^{(10)}$. The GSH concentration in blood basically accounts for its concentration in erythrocytes, blood concentration being about 40-fold that of plasma. The GSH concentration in erythrocytes depends only on the balance between its intracellular synthesis and the export of its oxidised form, as there are no known degradative pathways for GSH in erythrocytes and no efficient transport of the reduced form of GSH across their membrane ${ }^{(43)}$. Whatever the mechanism involved in the APAPinduced increase in blood GSH, the most interesting observation is its normalisation by Cys supplementation meaning that Cys deficiency plays a key role in APAP-induced increase in blood GSH. This is in agreement with the increased blood GSH already reported in methionine-restricted rats, also associated with decreases of liver GSH and plasma free Cys ${ }^{(44,45)}$. The increase in blood GSH appears to reflect the deficiency in Cys observed in APAP-treated rats. Thus, blood GSH may be helpful to determine the optimum Cys supplementation needed to prevent APAPinduced adverse metabolic outcomes.

APAP repeated cures decreased plasma protein-bound Cys-Gly and Hcy, and Cys supplementation compensated these effects to some extent. Aminothiols are either free or bound to proteins through disulphur bridges. Any increase in protein-bound forms without any modification in free forms reveals a modification of the redox status toward a more oxidative state. Thus, Cys supplementation prevented the pro-oxidative effect of repeated APAP cures. It also increased plasma albumin $v$. the CT group. As oxidative stress has been implicated in the pathogenesis of several ageing-associated pathologies ${ }^{(46,47)}$ and hypoalbuminemia is a mortality prognostic factor in elderly people ${ }^{(48)}$, it could be considered that Cys supplementation could have a real beneficial effect in elderly persons under chronic/repeated cures with APAP.

Muscle mass was not significantly reduced by repeated APAP cures, contrasting with our previous experiment performed with the same experimental design ${ }^{(11)}$. The difference in the susceptibility of old rats to APAP-induced negative effects between our two studies performed with the same experimental design could be attributed to the well-known inherent high variability between the cohorts of old rats ${ }^{(49)}$. That difference could not be attributed to a lower APAP dose really ingested by old rats as it reached $300 \mathrm{mg} / \mathrm{kg}$ per $\mathrm{d}$, a dose equivalent to a daily therapeutic dose of $3.5 \mathrm{~g} / \mathrm{d}$ for humans, whereas the APAP dose amounted to $260 \mathrm{mg} / \mathrm{kg}$ per $\mathrm{d}$ in the previous experiment ${ }^{(11)}$. In spite of a higher dose of APAP in the present study, no hepatotoxicity was recorded. Indeed, the present low variation in ALT is definitively below the threshold levels usually considered for hepatotoxicity, that is, three times the control values of AST and $\mathrm{ALT}^{(50)}$. Plasma concentrations of acute phase proteins revealed no APAP-induced inflammation. Consistently with these two observations, the low impact on protein synthesis in the liver, avoids competition between liver and muscle for the use of Cys. The main difference between our two studies was a food intake under APAP cures $12 \%$ higher in the second experiment than the first one ${ }^{(11)}$. This difference may explain why APAP-induced alterations in liver GSH, plasma free Cys (also named cyst(e)ine) and protein-bound Cys-gly and Hcy were presently light. Most importantly, these variations were not accompanied by a decline in plasma free GSH, whereas plasma free GSH was previously decreased with $\operatorname{APAP}^{(11)}$. The milder effect of APAP repeated cures on plasma free Cys associated with the absence of effect on plasma free GSH, a supplier of Cys, means that the decrease in the availability of Cys for the peripheral tissues, such as muscle, was definitively weak in the present study. It is likely that the present mild alteration in peripheral Cys/GSH homoeostasis was not sufficient to significantly affect muscle GSH and proteins. Altogether, the adverse metabolic outcomes induced by APAP in the liver and plasma appear to be milder when food intake is higher. These mild alterations were insufficient to impair muscle.

A typical design with APAP and Cys as experimental factors would have included a group of rats receiving the Cys supplementation without APAP and pair-fed to the three other 
experimental groups. In our previous experiment, a similar Cys supplementation induced only minor increases in Cys and GSH and no effect on muscle masses in old rats fed ad libitum $^{(34)}$. Thus, the conclusion of the present study would likely to have been unchanged by the inclusion of a group with a supplementation with Cys alone. Another limitation is that redox potentials (Eh) of the Cys/cystine and GSH/GSSG couples were not determined due to the technical choice to measure the total forms and the protein-bound forms and to calculate the free forms. However, knowing that the oxidised free form of GSH is always largely lower than its reduced form, the large present variations of either total or free forms can be confidently interpreted as significant variations in the reduced antioxidant form of GSH. In the same line of thought, oxidative stress was not deeply investigated in the present study because it is common knowledge that APAP treatment can induce oxidative stress consecutively to APAP-induced GSH decrease ${ }^{(51)}$. Thus, the present improvement in Cys/GSH homoeostasis in the APAP-Cys group can be considered with some certainty as a beneficial effect regarding oxidative stress.

In conclusion, sulphur amino acid requirement is increased by APAP and Cys can fulfil this demand. Indeed, dietary supplementation with Cys was efficient to improve Cys/GSH homoeostasis of old rats under repeated APAP cures. Cys supplementation exerted other beneficial effects to the liver and muscles that could have healthy outcomes. Moreover, Cys supplementation may be of further interest: (i) when a strong reduction of the peripheral availability of Cys/GSH occurs along with a generalised loss of muscle and (ii) for individuals susceptible to hepatotoxicity at therapeutic doses ${ }^{(14-16)}$. Finally, dietary Cys supplementation could be beneficial to the health of older persons under APAP treatment due to frequent low food intakes ${ }^{(52)}$ and the fact that their requirements in sulphur amino acids seem to be already higher than those of adults ${ }^{(53-55)}$.

\section{Acknowledgements}

The authors would like to acknowledge Medhi DjelloulMazouz, and Philippe Denis from the Installation Expérimentale de Nutrition for animal care.

This work was supported by the Institut National de la Recherche Agronomique, France and a Société francophone de nutrition clinique et métabolisme (SFNEP) - Antadir grant. SFNEP and Antadir had no role in the design, analysis or writing of this article.

The authors' contributions are as follows: I. P. and C. Mast designed the research; C. Mast, C. P. G. V., C. Migné and D. C. performed the experiment, C. Mast, C. P. and I. P. analysed the results, C. Mast and I. P. wrote the manuscript; I. S-A., D. R. and D. D. revised the manuscript; I. P. had primary responsibility for the final content. All authors read and approved the final manuscript.

The authors declare that there are no conflicts of interest.

\section{References}

1. Airaksinen O, Brox JI, Cedraschi C, et al. (2006) Chapter 4. European guidelines for the management of chronic nonspecific low back pain. Eur Spine J 15, S192-S300.
2. Nalamachu S (2013) An overview of pain management: the clinical efficacy and value of treatment. Am J Manag Care $\mathbf{1 9}$, s261-s266.

3. Makris UE, Abrams RC, Gurland B, et al. (2014) Management of persistent pain in the older patient: a clinical review. JAMA 312, 825-836.

4. Forrest JAH, Clements JA \& Prescott LF (1982) Clinical pharmacokinetics of paracetamol. Clin Pharmacokinet 7, 93-107.

5. Hodgman MJ \& Garrard AR (2012) A review of acetaminophen poisoning. Crit Care Clin 28, 499-516.

6. Obled C, Papet I \& Breuillé D (2004) Sulfur-containing amino acids and glutathione in diseases. In Metabolic and Therapeutic Aspects of Amino Acids in Clinical Nutrition, 2nd ed., pp. 667-687 [LA Cynober, editor]. Boca Raton, FL: CRC Press.

7. Pujos-Guillot E, Pickering G, Lyan B, et al. (2012) Therapeutic paracetamol treatment in older persons induces dietary and metabolic adaptations related to sulfur amino acids. Age 34, 181-193.

8. Nimni ME, Han B \& Cordoba F (2007) Are we getting enough sulfur in our diet? Nutr Metab (Lond) $\mathbf{4}, 24$.

9. Reicks M, Calvert RJ \& Hathcock JN (1988) Effects of prolonged acetaminophen ingestion and dietary methionine on mouse-liver glutathione. Drug Nutr Interact 5, 351-363.

10. Mast C, Joly C, Savary-Auzloux I, et al. (2014) Skeletal muscle wasting occurs in adult rats under chronic treatment with paracetamol when glutathione-dependent detoxification is highly activated. J Physiol Pharmacol 65, 623-631.

11. Mast C, Savary-Auzeloux I, Remond D, et al. (2016) Repeated cures with paracetamol worsen sarcopenia in old rats with suboptimal food intake. J Physiol Pharmacol 67, 759-768.

12. Jaeschke H (2015) Acetaminophen: dose-dependent drug hepatotoxicity and acute liver failure in patients. Dig Dis $\mathbf{3 3}$, 464-471.

13. Roberts E, Delgado Nunes V, Buckner S, et al. (2016) Paracetamol: not as safe as we thought? A systematic literature review of observational studies. Ann Rheum Dis 75, 552-559.

14. Amar PJ \& Schiff ER (2007) Acetaminophen safety and hepatotoxicity-where do we go from here? Expert Opin Drug Saf $\mathbf{6}$, 341-355.

15. O'Connell TM \& Watkins PB (2010) The application of metabonomics to predict drug-induced liver injury. Clin Pharmacol Ther 88, 394-399.

16. Winnike JH, Li Z, Wright FA, et al. (2010) Use of pharmacometabonomics for early prediction of acetaminophen-induced hepatotoxicity in humans. Clin Pharmacol Ther 88, 45-51.

17. Eriksson LS, Broome U, Kalin M, et al. (1992) Hepatotoxicity due to repeated intake of low doses of paracetamol. J Intern Med 231, 567-570.

18. Whitcomb DC \& Block GD (1994) Association of acetaminophen hepatoxicity with fasting and ethanol use. JAMA 272, 1845-1850.

19. Kurtovic J \& Riordan SM (2003) Paracetamol-induced hepatotoxicity at recommended dosage. J Intern Med $\mathbf{2 5 3}$, 240-243.

20. Kondo K, Yamada N, Suzuki Y, et al. (2012) Enhancement of acetaminophen-induced chronic hepatotoxicity in restricted fed rats: a nonclinical approach to acetaminophen-induced chronic hepatotoxicity in susceptible patients. J Toxicol Sci $\mathbf{3 7}$, 911-929.

21. Mitchell JR, Jollow DJ, Potter WZ, et al. (1973) Acetaminopheninduced hepatic necrosis. IV. Protective role of glutathione. J Pharmacol Exp Ther 187, 211-217.

22. Meister A (1991) Glutathione deficiency produced by inhibition of its synthesis, and its reversal; applications in research and therapy. Pharmacol Ther 51, 155-194. 
23. Prescott LF, Park J, Ballantyne A, et al. (1977) Treatment of paracetamol (acetaminophen) poisoning with $N$-acetylcysteine. Lancet $\mathbf{i i}, 432-434$.

24. Lauterburg BH, Corcoran GB \& Mitchell JR (1983) Mechanism of action of $N$-acetylcysteine in the protection against the hepatotoxicity of acetaminophen in rats in vivo. J Clin Invest 71, 980-991.

25. Owumi SE, Andrus JP, Herzenberg LA, et al. (2015) Co-administration of $N$-acetylcysteine and acetaminophen efficiently blocks acetaminophen toxicity. Drug Dev Res 76, 251-258.

26. Di Pierro F \& Rossoni G (2013) An amino acids mixture improves the hepatotoxicity induced by acetaminophen in mice. J Amino Acids 2013, 615754.

27. Kane AE, Huizer-Pajkos A, Mach J, et al. (2016) N-Acetyl cysteine does not prevent liver toxicity from chronic low-dose plus subacute high-dose paracetamol exposure in young or old mice. Fundam Clin Pharmacol 30, 263-275.

28. Obled C, Papet I \& Breuillé D (2002) Metabolic bases of amino acid requirements in acute diseases. Curr Opin Clin Nutr Metab Care 5, 189-197.

29. Mudd SH, Finkelstein JD, Irreverre F, et al. (1965) Transsulfuration in mammals. Microassays and tissue distributions of three enzymes of the pathway. J Biol Chem 240, 4382-4392.

30. Ishii I, Akahoshi N, Yu XN, et al. (2004) Murine cystathionine gamma-lyase: complete cDNA and genomic sequences, promoter activity, tissue distribution and developmental expression. Biochem J 381, 113-123.

31. Stipanuk MH \& Ueki I (2011) Dealing with methionine/ homocysteine sulfur: cysteine metabolism to taurine and inorganic sulfur. J Inherit Metab Dis 34, 17-32.

32. Breuillé D, Pouyet C, Malmezat T, et al. (1998) Differential effect of dietary supplementation with cysteine or cystine or methionine on liver glutathione concentration in septic rats. Clin Nutr 17, 66.

33. Breuillé D, Béchereau F, Buffière C, et al. (2006) Beneficial effect of amino acid supplementation, especially cysteine, on body nitrogen economy in septic rats. Clin Nutr 25, 634-642.

34. Vidal K, Breuille D, Serrant P, et al. (2014) Long-term cysteine fortification impacts cysteine/glutathione homeostasis and food intake in ageing rats. Eur J Nutr 53, 963-971.

35. Ferrell B, Argoff CE, Epplin J, et al. (2009) Pharmacological management of persistent pain in older persons. Pain Med 10, 1062-1083.

36. Muth-Selbach US, Tegeder I, Brune K, et al. (1999) Acetaminophen inhibits spinal prostaglandin E2 release after peripheral noxious stimulation. Anesthesiology 91, 231-239.

37. Savary-Auzeloux I, Magne H, Migné C, et al. (2013) A dietary supplementation with leucine and antioxidants is capable to accelerate muscle mass recovery after immobilization in adult rats. PLOS ONE 8, e81495.

38. Malmezat T, Breuillé D, Pouyet C, et al. (1998) Metabolism of cysteine is modified during the acute phase of sepsis in rats. J Nutr 128, 97-105.
39. Mayot G, Vidal K, Martin JF, et al. (2007) Prognostic values of alpha2-macroglobulin, fibrinogen and albumin in regards to mortality and frailty in old rats. Exp Gerontol 42, 498-505.

40. Reicks M \& Hathcock JN (1989) Prolonged acetaminophen ingestion in mice - effects on the availability of methionine for metabolic functions. J Nutr 119, 1042-1049.

41. Osborne-Pellegrin MJ \& Fau D (1992) Effects of chronic absorption of dietary supplements of methionine and cystine on arterial morphology in the rat. Exp Mol Pathol 56, 49-59.

42. Hannibal L \& Blom HJ (2017) Homocysteine and disease: causal associations or epiphenomenons? Mol Aspects Med 53, 36-42.

43. Srivastava SK (1977) Glutathione movements. In Membrane Transport in Red Cells, pp. 327-335 [JC Ellory and LL Lew, editors]. London, New York and San Francisco: Academic Press.

44. Richie JP, Leutzinger Y, Parthasarathy S, et al. (1994) Methionine restriction increases blood glutathione and longevity in F344 rats. FASEB J 8, 1302-1307.

45. Richie JP Jr, Komninou D, Leutzinger Y, et al. (2004) Tissue glutathione and cysteine levels in methionine-restricted rats. Nutrition 20, 800-805.

46. Manoharan S \& Guillemin GJ (2016) The role of reactive oxygen species in the pathogenesis of Alzheimer's disease, Parkinson's disease, and Huntington's disease: a mini review. Oxid Med Cell Longev 2016, 8590578.

47. Gomes MJ, Martinez PF, Pagan LU, et al. (2017) Skeletal muscle aging: influence of oxidative stress and physical exercise. Oncotarget 8, 20428-20440.

48. Cabrerizo S, Cuadras D, Gomez-Busto F, et al. (2015) Serum albumin and health in older people: Review and meta analysis. Maturitas $\mathbf{8 1}, 17-27$.

49. Ghirardi O, Cozzolino R, Guaraldi D, et al. (1995) Within- and between-strain variability in longevity of inbred and outbred rats under the same environmental conditions. Exp Gerontol 30, 485-494.

50. Temple R (2006) Hy's law: predicting serious hepatotoxicity. Pharmacoepidemiol Drug Saf 15, 241-243.

51. Jaeschke H, McGill MR \& Ramachandran A (2012) Oxidant stress, mitochondria, and cell death mechanisms in druginduced liver injury: lessons learned from acetaminophen hepatotoxicity. Drug Metab Rev 44, 88-106.

52. Calvani R, Miccheli A, Landi F, et al. (2013) Current nutritional recommendations and novel dietary strategies to manage sarcopenia. J Frailty Aging 2, 38-53.

53. Tuttle SG, Bassett SH, Griffith WH, et al. (1965) Further observations on the amino acid requirements of older men. II Methionine and lysine. Am J Clin Nutr 16, 229-231.

54. Mercier S, Breuillé D, Buffière C, et al. (2006) Methionine kinetics are altered in the elderly both in the basal state and after vaccination. Am J Clin Nutr 83, 291-298.

55. McCarty MF \& DiNicolantonio JJ (2015) An increased need for dietary cysteine in support of glutathione synthesis may underlie the increased risk for mortality associated with low protein intake in the elderly. Age (Dordr) 37, 96. 